University of Nebraska - Lincoln

DigitalCommons@University of Nebraska - Lincoln

\title{
The Importance of Fe Surface States For Magnetic Tunnel Junction Based Spintronic Devices
}

\author{
Athanasios N. Chantis \\ Los Alamos National Laboratory, achantis@lanl.gov \\ Kirill D. Belashchenko \\ University of Nebraska-Lincoln, belashchenko@unl.edu \\ Evgeny Y. Tsymbal \\ University of Nebraska-Lincoln, tsymbal@unl.edu \\ Inna V. Sus \\ Los Alamos National Laboratory
}

Follow this and additional works at: https://digitalcommons.unl.edu/physicsbelashchenko

Chantis, Athanasios N.; Belashchenko, Kirill D.; Tsymbal, Evgeny Y.; and Sus, Inna V., "The Importance of Fe Surface States For Magnetic Tunnel Junction Based Spintronic Devices" (2008). Kirill Belashchenko Publications. 7.

https://digitalcommons.unl.edu/physicsbelashchenko/7

This Article is brought to you for free and open access by the Research Papers in Physics and Astronomy at DigitalCommons@University of Nebraska - Lincoln. It has been accepted for inclusion in Kirill Belashchenko Publications by an authorized administrator of DigitalCommons@University of Nebraska - Lincoln. 


\title{
THE IMPORTANCE OF Fe SURFACE STATES FOR MAGNETIC TUNNEL JUNCTION BASED SPINTRONIC DEVICES
}

\author{
ATHANASIOS N. CHANTIS* \\ Theoretical Division, Los Alamos National Laboratory, \\ Los Alamos, New Mexico 87544, USA \\ achantis@lanl.gov
}

KIRILL D. BELASHCHENKO and EVGENY Y. TSYMBAL

Department of Physics and Astronomy and Nebraska Center for Materials and Nanoscience, University of Nebraska-Lincoln, Lincoln, Nebraska 68588, USA

\section{INNA V. SUS}

School of Materials, Arizona State University, Tempe, Arizona, 85287-6006, USA and Theoretical Division, Los Alamos National Laboratory, Los Alamos, New Mexico 87544, USA

\section{Received 14 September 2008}

\begin{abstract}
In this article we give a review of our recent theoretical studies of the influence of $\mathrm{Fe}(001)$ surface (interface) states on spin-polarized electron transport across magnetic tunnel junctions with Fe electrodes. We show that minority-spin surface (interface) states are responsible for at least two effects which are important for spin electronics. First, they can produce a sizable tunneling anisotropic magnetoresistance in magnetic tunnel junctions with a single Fe electrode. The effect is driven by a Rashba shift of the resonant surface band when the magnetization changes direction. This can introduce a new class of spintronic devices, namely, tunneling magnetoresistance junctions with a single ferromagnetic electrode. Second, in Fe/GaAs(001) magnetic tunnel junctions minority-spin interface states produce a strong dependence of the tunneling current spin polarization on applied electrical bias. A dramatic sign reversal within a voltage range of just a few tenths of an $\mathrm{eV}$ is predicted. This explains the observed sign reversal of spin polarization in recent experiments of electrical spin injection in $\mathrm{Fe} / \mathrm{GaAs}(001)$ and related reversal of tunneling magnetoresistance through vertical Fe/GaAs/Fe trilayers.
\end{abstract}

Keywords: Spin transport through interfaces; electrical injection of spin polarized carriers; spin polarized transport in semiconductors; spintronics; first principles electron transport methods; tunneling anisotropic magnetoresistance; metal-semiconductormetal structures; magnetic tunnel junctions; Rashba splitting.

\section{Introduction}

The spin-dependent properties of magnetic surfaces and interfaces have recently attracted a lot of attention because of the advent of spintronics, a technology aiming

*Corresponding author. 
to harness electron's spin in data storage and processing, typically by utilizing heterostructures composed of magnetic and non-magnetic materials. ${ }^{1}$ The search for a path to utilize spintronic devices in today's high-tech industry is often synonymous to the search for ways of injecting efficiently the electron spin into conventional semiconductors. A theoretical way was proposed independently by Smith et al. ${ }^{2}$ and Rashba et al., ${ }^{3}$ on how to generate non-equilibrium electron spin distributions in normal semiconductors with the help of ferromagnetic metal/non-magnetic semiconductor contacts. Among various ferromagnet/semiconductor structures, $\mathrm{Fe} / \mathrm{GaAs}$ contacts have been extensively studied, and efficient electrical spin injection by tunneling through Schottky barriers formed by delta doping has been achieved by many groups. ${ }^{4-8}$ The spin polarization in these experiments originates from the spin dependence of the wavefunctions and densities of states of the Fe contact. As a result, the tunneling transmission coefficients are different for majorityand minority-spin electrons. The experiments by Habincki et al. ${ }^{5,6}$ and Adelman et al. ${ }^{7}$ showed that the net polarization of injected current is parallel to the majorityspin in Fe, which is what is naturally expected from the density of states of any bulk ferromagnet. However, the most recent experiments by Crooker et al. ${ }^{4}$ and Lou et $a l .{ }^{8}$ have shown that the spin polarization of injected or accumulated electrons at the $\mathrm{Fe} / \mathrm{GaAs}(001)$ interface can be either of majority or minority-spin character. In particular, the second experiment ${ }^{8}$ showed that the spin polarization depends strongly on applied bias voltage producing either majority- or minority-spin accumulation in GaAs within a small range of a few tenths of an $\mathrm{eV}$. In a related experiment, Moser et al. ${ }^{14}$ observed that in Fe/GaAs/Fe magnetic tunnel junctions the tunneling magnetoresistance (TMR) changes sign with bias voltage. The TMR changed sign only when one of the interfaces was epitaxial, reflecting the reversal of the spin polarization at the epitaxial Fe/GaAs interface.

The electronic structure of transition metal surfaces is expected to exhibit localized surface states formed by dangling $d$ bonds. Once these localized surface states enter the bulk continuum, hybridization with bulk bands forms narrow interface resonant bands. ${ }^{39}$ The existence of at least one such band was confirmed experimentally for $\mathrm{Fe}(001)$ by scanning tunneling microscopy (STM) in Ref. 12 (STM in other transition metals shows similar features $\left.{ }^{12}\right)$. The current-voltage characteristic of the STM in Ref. 12 shows that this band is located in the vicinity of Fermi energy. Recent spin-resolved photoemission spectroscopy of Fe(001) surface shows that this band is of minority-spin, ${ }^{37}$ in agreement with previous spin-resolved and angle-resolved photoemission spectroscopy (SARPES) ${ }^{11}$ and $a b$ initio calculations. ${ }^{10}$ (The latter attributes the surface states to minority-spin $d$-orbitals.) There is experimental evidence that interface states also exist in $\mathrm{Fe} / \mathrm{MgO}^{37,38}$ which may have significant implications for transport properties. ${ }^{40}$

Another important effect at the transition metal surface/interfaces is the increased role of spin-orbit interaction (SOI) due the surface/interface Rashba effect. ${ }^{9}$ The spin-orbit interaction is responsible for the magnetic anisotropy and spin-mixing of electron states. Both effects can be important for spin-dependent 
tunneling, especially with respect to the prospect of novel spintronic devices that are based on a tunneling magnetoresistance effect without the need for non-colinear configuration between different ferromagnetic layers. This prospect has appeared recently with the discovery of tunneling anisotropic magnetoresistance (TAMR) in tunnel junctions that contain only one ferromagnetic contact. ${ }^{13}$ TAMR is the change in the electric conductance of the junction when the orientation of applied magnetic field changes from perpendicular to parallel to the interface. It is entirely based on the SOI of the ferromagnetic layer. Unfortunately, the ferromagnetic layer in these experiments was diluted GaMnAs which has low transition temperatures and therefore cannot be used in real-world spintronic devices. Since the appearance of this discovery, the search for paths towards achieving TAMR at higher temperatures has been a topic of great interest. Until now, the consequences of the surface Rashba SOI and the Fe surface states on spin-dependent transport across magnetic metal junctions of Fe have not been fully addressed.

Here, we give a brief summary of our related studies with the help of a fully relativistic $a b$ initio technique. The results indicate that minority-spin interface bands in $\mathrm{Fe} / \mathrm{GaAs}(001)$ are responsible for the observed dramatic bias dependence of current spin polarization. We also demonstrated that the Rashba SOI shifts the $\mathrm{Fe}(001)$ surface states when the magnetization changes direction producing high TAMR ratios. This shows a way of achieving TAMR at room temperatures by utilizing $\mathrm{Fe} /$ semiconductor junctions.

\section{Method}

In this section, we give a brief description of the numerical method used in the study. The method is based on the Green's function representation of the tight-binding linear muffin-tin orbital (TB-LMTO) basis in the atomic spheres approximation. ${ }^{15}$ Within the relativistic formulation of the local spin density approximation (LSDA) in which only the spin component of the current density is taken into account, ${ }^{16}$ inside each atomic sphere we solve the Kohn-Sham Dirac equation ${ }^{17}$

$$
\left[c \mathbf{a p}+(\beta-I) m c^{2}+V(r)+\mu_{B} B(r) \mathbf{n} \boldsymbol{\Sigma}\right] \Psi(E, \mathbf{r})=E \Psi(E, \mathbf{r}),
$$

where

$$
\alpha=\left(\begin{array}{cc}
0 & \boldsymbol{\sigma} \\
\boldsymbol{\sigma} & 0
\end{array}\right), \quad \beta=\left(\begin{array}{rr}
I & 0 \\
0 & -I
\end{array}\right), \quad \Sigma=\left(\begin{array}{ll}
\boldsymbol{\sigma} & 0 \\
0 & \boldsymbol{\sigma}
\end{array}\right) .
$$

Here, $\boldsymbol{\sigma}$ is the vector of Pauli matrices, $\mathbf{p}$ is the momentum operator, and $\mathbf{n}$ is the unit vector in the direction of the effective magnetic field inside the MT sphere. The energy $E$ is referenced to the total relativistic energy $W=m c^{2}+E$. The effective magnetic field in Eq. (1) can be found $\operatorname{as}^{18} B(r)=\left(V^{\uparrow}(r)+V^{\downarrow}\right)(r) / 2$. The solutions of the Kohn-Sham Dirac equation are linear combinations of bispinors:

$$
\Psi_{\mu}(\mathbf{r}, E)=\sum_{\kappa} \Psi_{\kappa \mu}(\mathbf{r}, E),
$$




$$
\Psi_{\kappa \mu}(\mathbf{r}, E)=\left(\begin{array}{c}
g_{\kappa \mu}(E, r) \Omega_{\kappa \mu}(\hat{r}) \\
i f_{\kappa \mu}(E, r) \Omega_{-\kappa \mu}(\hat{r})
\end{array}\right) .
$$

Here, $\Omega_{\kappa \mu}(\hat{r})$ are the spin spherical harmonics, $\mu$ is the projection of the total angular momentum and $\kappa$ is the relativistic quantum number: $\kappa^{2}=J(J+1)+\frac{1}{4}$.

Within the fully relativistic LMTO method, the boundary conditions at the MT radius $s$ are given in the form of two matrix equations ${ }^{19,21}$

$$
\begin{aligned}
& N(E)=(2 l+1)\left(\frac{w}{s}\right)^{l+1} g^{-1}(E, s)(D(E, s)-I l)^{-1}, \\
& P(E)=2(2 l+1)\left(\frac{w}{s}\right)^{2 l+1}(D(E, s)+I l+I)(D(E, s)-I l)^{-1},
\end{aligned}
$$

where $N(E), P(E), D(E)$ and $g(E)$ are $2 \times 2$ matrices for each value of $\kappa, \mu$ and site $R . N(E)$ and $P(E)$ are arbitrary matrices defined by the boundary conditions, with elements of the $\kappa, \mu$ components of the so-called potential and normalization functions defined in the scalar relativistic LMTO method. ${ }^{20} D(E, s)$ and $g(E, s)$ are matrices with elements of the $\kappa, \mu$ components of logarithmic derivative and large component of the wavefunction. The primary difference of this work with previous fully relativistic formulations of LMTO method is that we use the third-order parametrization of the potential functions ${ }^{22,23}$ : the radial amplitudes are expanded up to quadratic terms in linearization energy $\epsilon_{\nu}$,

$$
\Psi_{\kappa \mu}(E, \mathbf{r})=\Psi_{\kappa \mu}^{\nu}(\mathbf{r})+\left(E-\epsilon_{\nu}\right) \dot{\Psi}_{\kappa \mu}^{\nu}(\mathbf{r})+\frac{1}{2}\left(E-\epsilon_{\nu}\right)^{2} \ddot{\Psi}_{\kappa \mu}^{\nu}(\mathbf{r})
$$

The Green's function of the layered system is constructed by the principal-layer technique. ${ }^{19}$ The layers from $-\infty$ to 0 and from $N+1$ to $\infty$ are the contacts, while the layers from 1 to $N$ are the active layers. Then, the transmission coefficient within the Landauer-Büttiker approach ${ }^{24}$ can be calculated as

$$
\begin{aligned}
T\left(k_{\|}, E\right)= & \lim _{\epsilon \rightarrow 0^{+}} \frac{1}{2} \operatorname{Tr}\left[B_{1}\left(k_{\|}, E\right) g_{1, N}\left(k_{\|}, z_{+}\right) B_{N}\left(k_{\|}, E\right) g_{N, 1}\left(k_{\|}, z_{-}\right)\right. \\
& \left.+B_{1}\left(k_{\|}, E\right) g_{1, N}\left(k_{\|}, E\right) g_{N, 1}\left(k_{\|}, z_{+}\right)\right]
\end{aligned}
$$

where

$$
B_{p}\left(k_{\|}, E\right)=i\left[\Gamma_{p}\left(k_{\|}, z_{+}\right)-\Gamma_{p}\left(k_{\|}, z_{-}\right)\right],
$$

with $p=1, N, z_{ \pm}=E \pm i \epsilon$ and

$$
\Gamma_{p}\left(k_{\|}, E\right)= \begin{cases}S_{1,0}\left(k_{\|}\right) G_{L}^{s f}\left(k_{\|}, z\right) S_{0,1}\left(k_{\|}\right) ; & p=1, \\ S_{N, N+1}\left(k_{\|}\right) G_{R}^{s f}\left(k_{\|}, z\right) S_{N+1, N}\left(k_{\|}\right) ; & p=N .\end{cases}
$$

The surface Green's functions of the electrodes $G_{L, R}^{s f}\left(k_{\|}, z\right)$ are constructed scalarrelativistically, which allows us to decompose the conductance into spin-conserving 
and spin-flip components. ${ }^{25} g_{1, N}$ and $g_{N, 1}$ are the upper-right corner and lower-left corner components of the auxiliary green's function matrix

$$
g=[P-S]^{-1} \text {. }
$$

Here, $P$ is the fully relativistic potential function (6) and $S$ the tridiagonal matrix of scalar relativistic structure constants. ${ }^{20}$

\section{Results}

\section{1. $\mathrm{Fe}(001)$ surface}

In our calculations, we use a set-up representing an $\mathrm{Fe}(001) /$ vacuum/ $\mathrm{Cu}$ tunnel junction. The $\mathrm{Cu}$ electrode has a spin-independent free-electron-like band structure and a featureless surface transmission function, ${ }^{26}$ which makes it insensitive to the transverse wavevector. This electrode simulates either an ideal STM tip or an idealized second electrode in the TAMR device. Another advantage of such setup is that it avoids the possible "matching" of surface resonances at two opposite metallic surfaces/interfaces. ${ }^{27}$ When two resonant states on opposite contacts have the same energy and $k_{\|}$, then a resonant transmission will occur across the structure. Obviously a situation like this may occur in a symmetric structure. However, in real structures such "handshakes" are unlikely because the symmetry of the structure is broken by applied bias or disorder. Therefore, a Fe(001)/vacuum/Fe(001) setup may yield unphysical high transmission for surface states. The semi-infinite Fe and $\mathrm{Cu}$ electrodes are separated by approximately $1 \mathrm{~nm}$ of vacuum represented by 6 monolayers of empty spheres. Self-consistent charge distribution is achieved using separate scalar-relativistic TB-LMTO calculations for $\mathrm{Fe}$ and $\mathrm{Cu}$ surfaces treated using supercells with 12 metallic monolayers. The conductance calculations are performed at zero bias using a uniform $200 \times 200$ mesh in the surface Brillouin zone.

The spin-dependent transmission coefficient $t^{\sigma}\left(E, k_{\|}\right)$is calculated for a given spin $\sigma=\uparrow, \downarrow$ (where $\uparrow$ and $\downarrow$ denote majority and minority spin, respectively) as a function of energy $E$ and the transverse wave vector $k_{\|}$which is conserved due to the transverse periodicity of the junction. The total transmission for a given energy and spin is obtained by integrating over $k_{\|}$within a two-dimensional Brillouin zone (2DBZ):

$$
T^{\sigma}(E)=\left(\frac{1}{2 \pi}\right)^{2} \int t^{\sigma}\left(E, k_{\|}\right) d^{2} k_{\|} .
$$

A uniform $200 \times 200$ mesh is used for the integration. The current density associated with this transmission is obtained from

$$
J^{\sigma}(V)=\frac{e}{h} \int_{E_{F}}^{E_{F}+e V} T^{\sigma}(E) d E,
$$




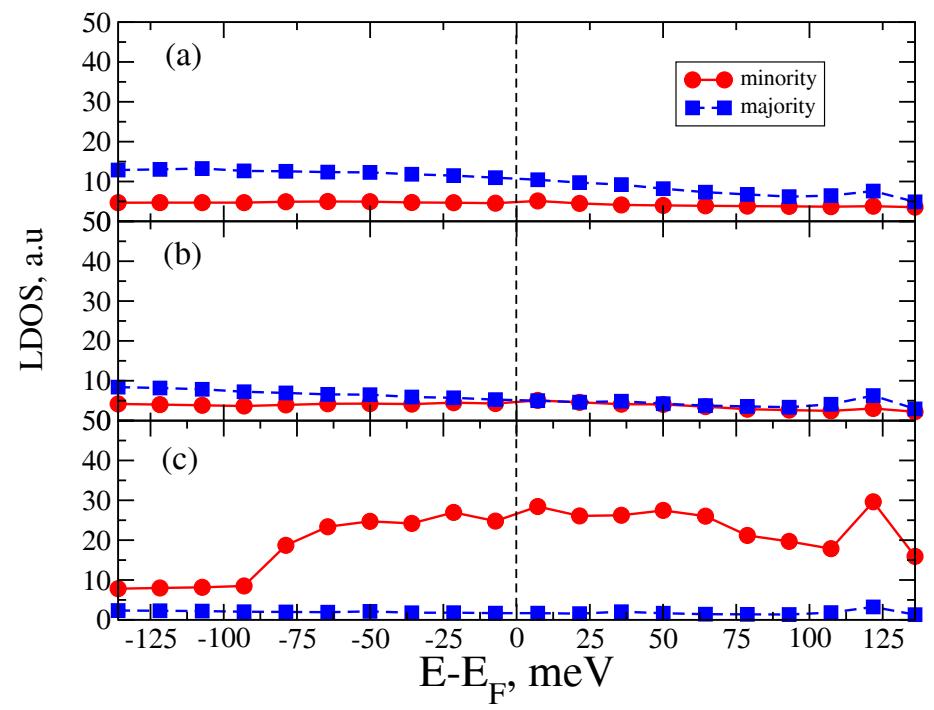

Fig. 1. Spin-resolved layer DOS of Fe(001) in the vicinity of Fermi energy, red line (minorityspin), blue line (majority-spin): (a) Bulk Fe; (b) Fe subsurface layer; (c) Fe surface layer.

where $E_{F}$ is the Fermi energy and $V$ is the applied bias voltage. This is a reasonable approximation for the small voltages considered in this work. This definition of $J^{\sigma}(V)$ implies that for a negative voltage electrons tunnel from Fe across GaAs. The spin polarization is defined as

$$
P=\frac{\left(J^{\uparrow}-J^{\downarrow}\right)}{\left(J^{\uparrow}+J^{\downarrow}\right)} .
$$

In Fig. 1, we present the scalar relativistic spin-resolved Layered Density of States (LDOS). Figure 1(a) is the DOS in bulk Fe, Fig. 1(b) in the subsurface monolayer and Fig. 1(c) in the surface monolayer. In the bulk, the majority-spin dominates over the minority-spin throughout the entire energy interval shown here. However, as shown in Fig. 1(c), in the surface layer, it is the minority-spin that dominates. In the subsurface layer, the strong minority-spin features of the DOS have already decayed and the bulk picture is almost recovered. This is a clear signature of localized surface states. The finite width of the DOS minority spin peak at the surface indicates that the surface states form a narrow surface band due to partial overlap with the minority spin Fe bulk band. Since most of the electrons that contribute to the tunneling current originate from the vicinity of the Fermi surface, one would expect that this sign reversal could have a significant impact on the polarization of the tunneling current. However, sign reversal of spin polarization of local DOS does not necessarily lead to sign reversal of the spin polarization of tunneling transmission, at least throughout the same energy interval. Moreover, it is even less clear if it leads to a reversal of the spin polarization of the tunneling current within the voltage range that corresponds to the shown electron energies. 

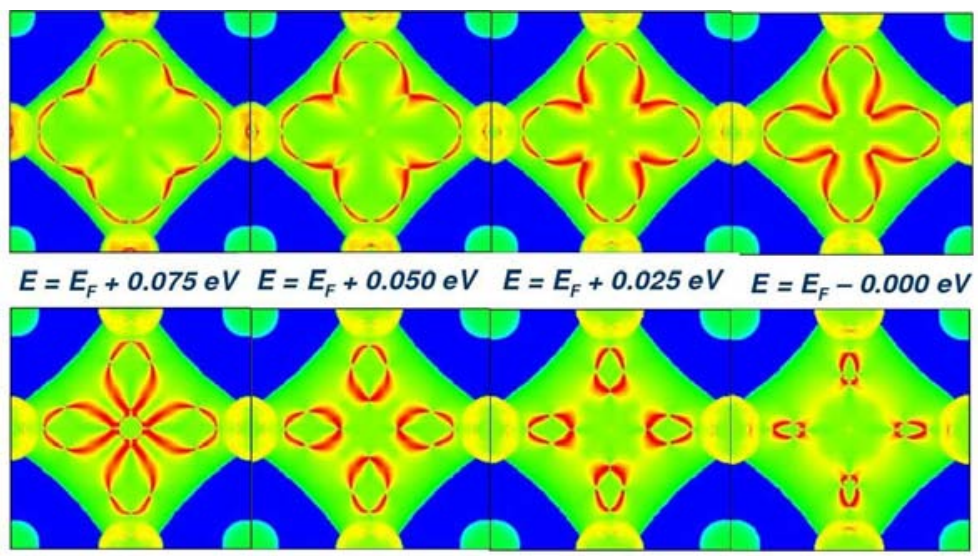

$E=E_{F}-0.025 \mathrm{eV} \quad E=E_{F}-0.050 \mathrm{eV} \quad E=E_{F}-0.075 \mathrm{eV} \quad E=E_{F}+0.100 \mathrm{eV}$

Fig. 2. Minority-spin $k_{\|}$-resolved DOS of the Fe(001) surface for different energies around the Fermi level. The abscissa is along [100] and the ordinate is along [010]. The largest value is represented by red color, the lowest by blue.

In Fig. 2, we present the minority-spin $k_{\|}$-resolved DOS of the Fe(001) surface for different energies around the Fermi level. The bright red features on these plots are created by the surface band. Unlike the majority-spin bulk band, this band never crosses the $2 \mathrm{DBZ}$ at the $\Gamma$ point. The closest it gets to the $\Gamma$ point is at the energy of $E_{F}=0.025 \mathrm{eV}$ where we see a bright four petal structure centered at $\Gamma$ point without touching it. Considering that $k_{\|}$is conserved during tunneling across an ideal surface, for an electron entering the vacuum region with a real $k_{\|}$, the electron wavefunction decay rate is proportional to $\exp \left[-\left(\kappa^{2}+k_{\|}^{2}\right) z\right]$, where $z$ is the distance and $\kappa$ is the decay rate for normal incidence, determined by the potential height of the tunneling barrier. The tunneling transmission for a given energy is also proportional to the total number of states $n(E)$ at this energy. In Fig. 3, one can compare the spin-resolved tunneling transmission with the spinresolved surface DOS. We see the influence of both factors just mentioned above on the polarization of the transmission coefficient. (For better comparison, the spin polarization of DOS, transmission and current density are shown all together in Fig. 4.) The minority-spin transmission dominates over the majority-spin for a large part of the energy interval due to its much higher surface DOS. For the energy $E=E_{F}-0.025 \mathrm{eV}$, the minority-spin transmission has a maximum and in the energy interval where the minority-spin DOS is flat, the minority-spin transmission decreases as the surface states move away from the $\Gamma$ point. For energies where the minority-spin surface states are far away from the center of the 2DBZ, the transmission from the minority-spin is less than that from the majority-spin even though for the same energy the surface DOS of the minority-spin is much larger than that of the majority-spin. The tunneling current density is an integrated property over all electron energies between $E_{F}$ and $E_{F}+\mathrm{eV}$ (Fig. 3(c)). 


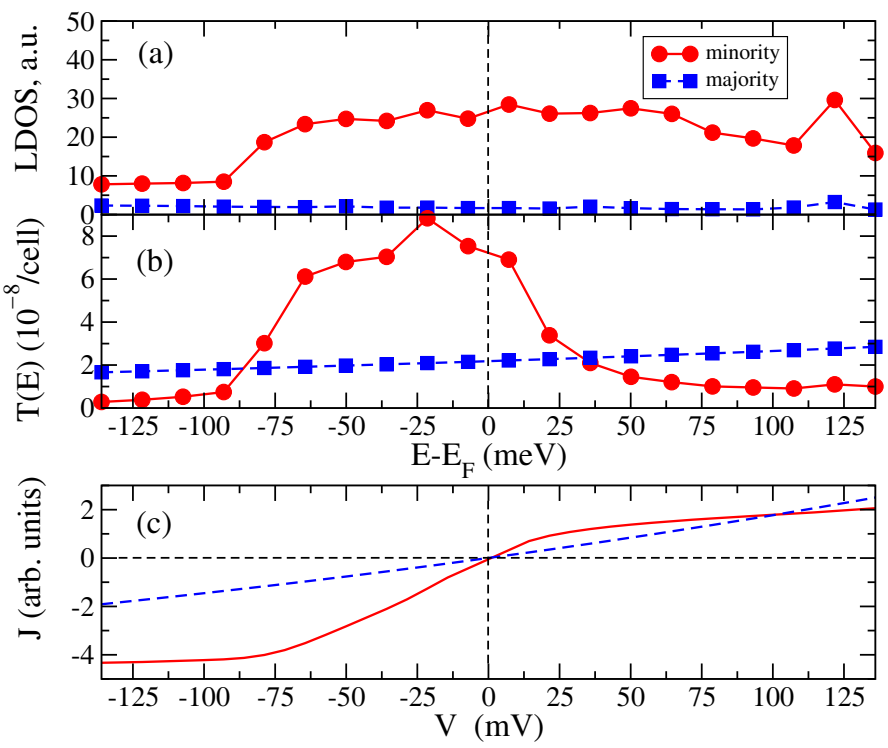

Fig. 3. (a) Spin-resolved DOS of the Fe(001) surface, (b) spin-resolved $k_{\|}$-integrated transmission $T$ for the $\mathrm{Fe}(001)$ surface with a $\mathrm{Cu}$ counterelectrode placed at $8.6 \AA$ from the surface, (c) the spin-resolved current density. In all panels the red solid line corresponds to the minority-spin, and the blue dashed line to the majority-spin.
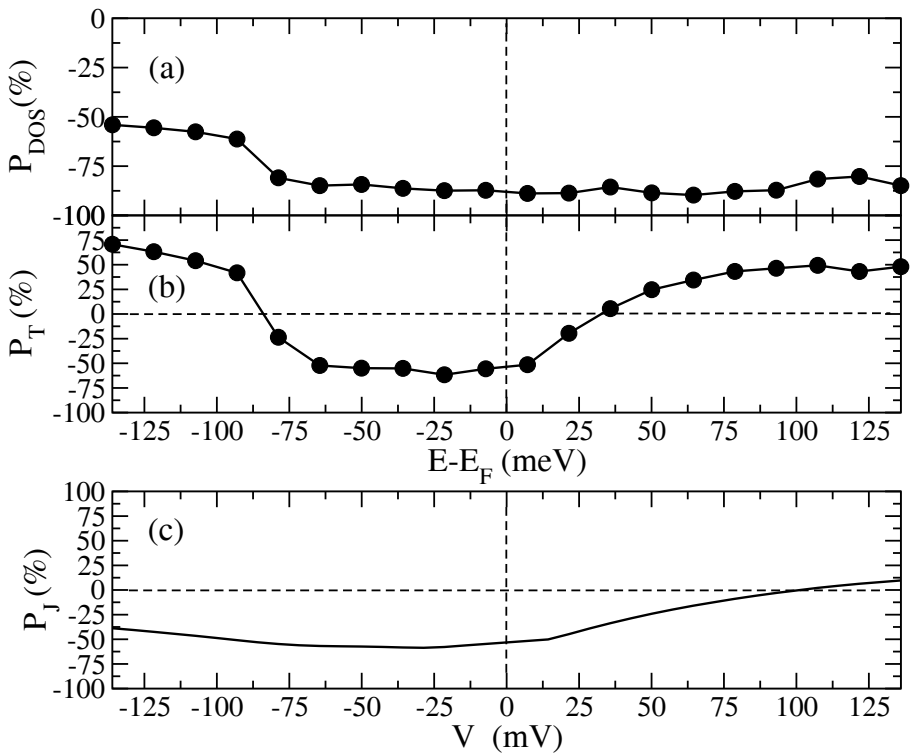

Fig. 4. Spin polarization of (a) DOS, (b) transmission, (c) current density for Fe(001). Negative polarization means that the minority-spin dominates. The transmission and current density are calculated with a $\mathrm{Cu}$ counterelectrode placed at $8.6 \AA$ from the surface. 
We see that the large minority-spin peak of the transmission around $E_{F}$ results in a minority-spin current polarization throughout almost the entire voltage range shown in Fig. 3(c). However, one should take into account that for a bigger separation between the Fe surface and $\mathrm{Cu}$ counterelectrode both the height and width of the minority-spin peak of the transmission should become smaller relative to the majority spins. Consequently, both $P_{T}$ and $P_{J}$ will vary with distance acquiring more majority character as the distance increases. However, our calculations show that for a distance twice as big, the change of spin polarization is not very big while the current drops by about four orders of magnitude. This shows that within the range of distances available to an STM ferromagnetic tip, the inversion of spin polarization should be detectable. ${ }^{41}$

\subsection{Tunneling anisotropic magnetoresistance}

The SOI can alter the electronic structure of bulk materials and induce magnetic anisotropy. It is also responsible for the spin-flip part of electron scattering. The strength of bulk SOI is proportional to the atomic number $Z^{2}$, and its influence depends on the atomic character of the band. For example a fully relativistic band structure calculation for $G d$ shows overall much stronger SOI corrections to the scalar relativistic calculation than it does for $\mathrm{Fe},{ }^{28}$ especially for the $\mathrm{Gd} 4 f$ orbitals. Nevertheless, in Fe depending on the direction of the magnetization, SOI induces band splittings and opens several band gaps along the $\Gamma-\Delta-H$ line, in the vicinity of $E_{F}$. It was shown in Ref. 27 that some of this band gaps can have a weak influence on the transmission but overall they cannot be responsible for a sizable TAMR. Overall, one cannot expect significant SOI effects in Fe from this effect alone.

However, in metallic surfaces and interfaces due to intrinsic structural asymmetry, SOI can demonstrate itself in the form of a Rashba effect. ${ }^{9}$ The Rashba effect can be interpreted as an effective $\mathbf{k}$-dependent magnetic field seen by the surface electron and is described by the Hamiltonian:

$$
H_{R}=\alpha\left[\mathbf{e}_{\mathbf{z}} \times \mathbf{k}\right] \mathbf{s},
$$

where the constant $\alpha$ depends on the strength of the electric field at the surface/interface and material-dependent parameters, $\mathbf{e}_{\mathbf{z}}$ is the unit vector perpendicular to the surface, $\mathbf{k}$ is the electron wave vector and $\mathbf{s}$ the electron spin-vector. In non-magnetic systems, this operator results in a $k$-space isotropic spin-split of the electron energy bands. However, in magnetic systems, the spin degeneracy of the bands is already lifted by the exchange interaction. Let us examine the effect of this operator on a spin-polarized electron. Equation (15) can be written as

$$
H_{R}=\frac{\hbar}{2} \alpha\left(k_{y} \sigma_{x}-k_{x} \sigma_{y}\right) .
$$

The magnetization defines a spin-quantization axis. If we direct the magnetization along the(001) direction $\left(\mathbf{s} \| k_{z}\right)$, then it is easy to see that the above operator does 

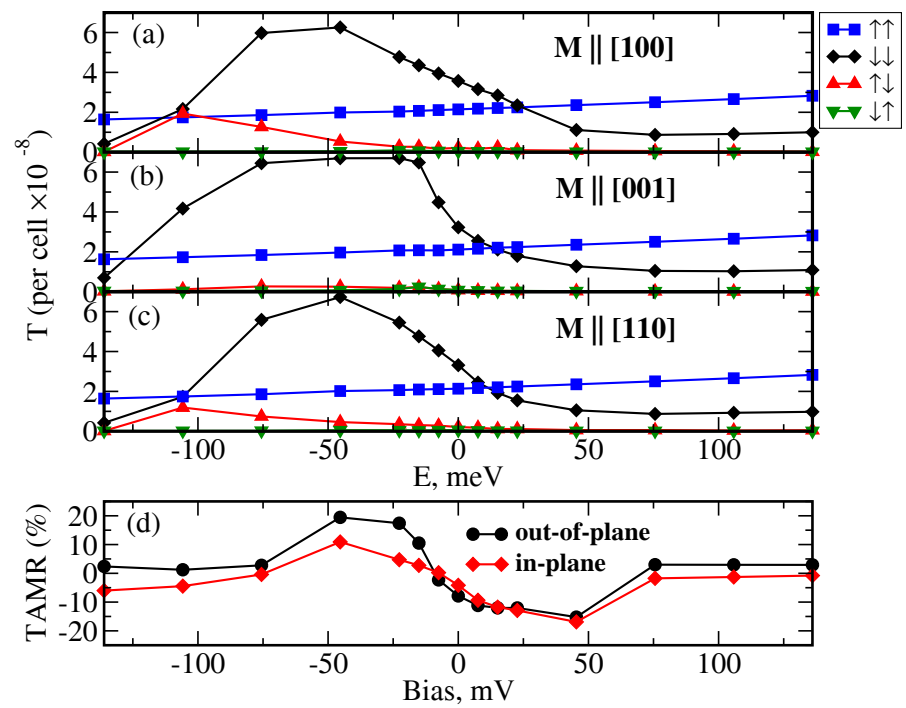

Fig. 5. Spin-resolved integrated transmission $T_{\sigma \sigma^{\prime}}$ for the $\mathrm{Fe}(001)$ surface with a $\mathrm{Cu}$ counterelectrode as a function of energy. Magnetization is along (a) [100], (b) [110], and (c) [001] directions. The Fermi level is at zero energy. (d) In-plane and out-of-plane TAMR as a function of bias voltage.

not act on the spin-component of the wavefunction and therefore has no influence on the electron bands. If we direct the magnetization in plane, for example along (010), then the energy bands of a spin-polarized free electron are the eigenvalues of the Hamiltonian:

$$
H=\left(\begin{array}{cc}
\frac{\hbar^{2} k^{2}}{2 m}+\frac{\Delta}{2} & -i \frac{\hbar}{2} \alpha k_{x} \\
i \frac{\hbar}{2} \alpha k_{x} & \frac{\hbar^{2} k^{2}}{2 m}-\frac{\Delta}{2}
\end{array}\right)
$$

where $\Delta$ is the exchange splitting. Along $(010),\left(k_{\|}|| \mathbf{s}\right) k_{x}=0$ and the Rashba term vanishes, and only the exchange splitting of the bands remains. For any other direction, the Rashba interaction shifts each of the exchange split bands. Even though the shift should be much less than the exchange splitting, it can have a visible effect on each band separately when its magnitude is comparable to the bandwidth.

In Figs. 5(a)-5(c), we show $T_{\sigma \sigma^{\prime}}$ for three magnetization directions. The energy dependence represents the linear-response conductance in the rigid-band model, which approximately reflects the effects of alloying. It is seen that $T_{\uparrow \uparrow}$ exhibits featureless free-electron-like energy dependence. However, $T_{\downarrow \downarrow}$ is non-monotonic and dominates in the energy range between $-125 \mathrm{meV}$ and $25 \mathrm{meV}$. The TAMR $\operatorname{ratios}\left(T^{\hat{\mathbf{n}}}-T^{[\mathbf{1 0 0}]}\right) / T^{[100]}$ are shown in Fig. $5(\mathrm{~d})$ for both out-of-plane $(\hat{\mathbf{n}}=[001])$ 

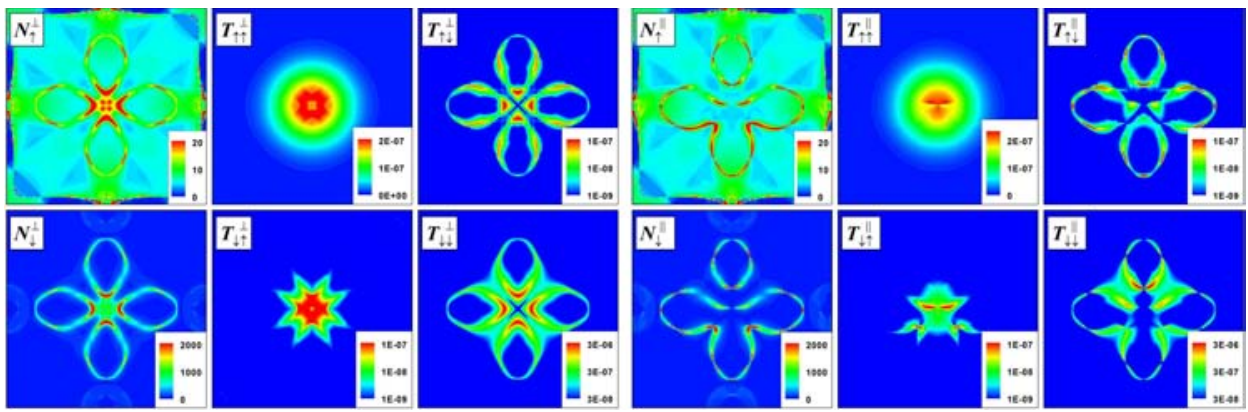

Fig. 6. Spin components of the DOS $N_{\sigma}$ at the Fe(001) surface monolayer and the transmission functions $T_{\sigma \sigma^{\prime}}$ for the $\mathrm{Fe}(001)$ surface with a $\mathrm{Cu}$ counterelectrode at $50 \mathrm{meV}$ below $E_{F}$. Figures are resolved by $k_{\|}$with abscissa along [100] and ordinate along [010]. The left six panels correspond to the magnetization normal to the surface (labeled by superscript $\perp$ ), and the right half to the in-plane magnetization aligned along [100] (labeled by superscript $\|$ ). Some panels are given in a logarithmic scale.

and in-plane $(\hat{\mathbf{n}}=[110])$ magnetization orientations as a function of bias voltage. ${ }^{\text {a }}$ In both cases, the TAMR has a strong bias dependence with a spectacular change of sign close to the Fermi level and a maximum/minimum of $\pm 15-20 \%$ at the bias voltage of $\pm 50 \mathrm{mV}$.

To understand the physical origin of large TAMR effect, we focus on energy $-50 \mathrm{meV}$ where the conductance has the strongest spin asymmetry. Figure 5 shows spin- and $k_{\|}$-resolved surface densities of states (DOS) and tunneling transmission functions. The left six panels correspond to the out-of-plane magnetization $(\hat{\mathbf{n}}=[001])$, and the right half to the in-plane magnetization $(\hat{\mathbf{n}}=[100])$. The resonant surface band is responsible for the bright "four-petal-flower" features in the minority-spin surface DOS $\left(N_{\downarrow}^{\perp}\right.$ and $\left.N_{\downarrow}^{\|}\right)$and in the minority-spin transmission $\left(T_{\downarrow \downarrow}^{\perp}\right.$ and $\left.T_{\downarrow \downarrow}^{\|}\right)$. This band is dominated by the minority-spin surface states, which mix weakly with bulk bands. SOI can strongly enhance this mixing, in both spin-diagonal and spin-mixing components. In particular, consider the surface state lying on the $\bar{\Gamma} \bar{X}$ line with $k_{\|}=\left(k_{x}, 0\right)$. In the absence of SOI, these eigenstates have definite parity with respect to reflection in the $y=0$ plane. The surface band is even, while the minority-spin bulk band is odd. By symmetry, these states cannot mix, and the surface state remains localized. The SOI does not conserve this parity and mixes the surface state with both minority-spin and majority-spin bulk states. The surface state is thus transformed into a surface resonance. In our case, this occurs at special $k_{\|}$points. In general, if for a given spin the surface band lies within a gap of bulk bands, the SOC converts this localized surface band into a resonant band.

As is evident from Fig. 6, the surface bands depend on spin orientation (compare $N_{\uparrow}^{\perp}$ to $N_{\uparrow}^{\|}$and $N_{\downarrow}^{\perp}$ to $\left.N_{\downarrow}^{\|}\right)$. This can be attributed to the Rashba effect described

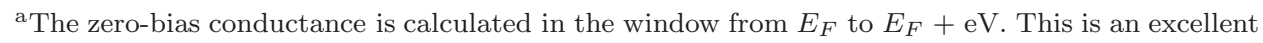
approximation appropriate for comparison with STS, because the considered voltages are small. 
above. The effective spin-orbit shift of electron energy is given by

$$
\Delta \epsilon\left(k_{\|}\right)=\alpha\left(\hat{\mathbf{z}} \times k_{\|}\right) \cdot \mathbf{s},
$$

where $\hat{\mathbf{z}}$ is the unit vector normal to the surface. The electron spin $\mathbf{s}$ is aligned with the magnetization $\mathbf{M}$. For $\mathbf{M} \| \hat{\mathbf{z}}$, the Rashba shift is zero throughout the surface Brillouin zone, while for $\mathbf{M} \| \hat{\mathbf{x}}$ it is positive for $k_{y}>0$ and negative for $k_{y}<0$. The asymmetric shift of the surface bands is reflected in the loss of fourfold symmetry in the right six panels of Fig. 6, and in particular in the loss of mirror symmetry in $y$. A similar effect was discussed for the $\operatorname{Gd}(0001)$ surface ${ }^{36}$ For $\mathbf{M} \| \hat{\mathbf{z}}$, a portion of the resonant surface band lies close to the $\bar{\Gamma}$ point and adds a large contribution to the minority-spin conductance. When the magnetization is rotated to $\mathbf{M} \| \hat{\mathbf{x}}$ these states shift, and the conductance is reduced. This is the origin of the large TAMR effect seen in Fig. 5. Another noticable feature on these plots has to do with the spin-flip components. Namely, comparison of $N_{\uparrow}^{\perp}$ to $N_{\downarrow}^{\perp}$, as well as $N_{\uparrow}^{\|}$to $N_{\downarrow}^{\|}$, in Fig. 6 indicates that the admixture of majority-spin states to the surface band is of the order of $1 \%$ (note the difference in scales). However, the spin-flip components of the transmission are quite pronounced. For example, in certain areas of the Brillouin zone, both $T_{\uparrow \downarrow}$ and $T_{\downarrow \uparrow}$ are comparable to the majority-spin component $T_{\uparrow \uparrow}$. We will discuss this issue in great detail in the following subsection.

\subsection{Resonant spin-flip transmission}

The spin-flip components of the transmission function $T_{\uparrow \downarrow}$ and $T_{\downarrow \uparrow}$ shown in Fig. 5 display a non-monotonic energy dependence and are generally quite small compared to the spin-conserving components. Surprisingly, for the in-plane magnetization, $T_{\uparrow \downarrow}$ has a pronounced maximum at $E_{F}-0.1 \mathrm{eV}$, just above the bottom of the resonant surface band, which extends to higher energies (Fig. 5(a)). Here, the spinflip and spin-conserving contributions are comparable. Notably, the peak appears only for the in-plane magnetization, and the spin-flip process is strongly asymmetric: $T_{\uparrow \downarrow} \gg T_{\downarrow \uparrow}$. Figure 7 shows $k_{\|}$-resolved spin-flip transmission function $T_{\uparrow \downarrow}\left(k_{\|}\right)$for both magnetization orientations. The resonant surface bands are seen as four small ellipses along the $\bar{\Gamma} \bar{X}$ lines. All the difference in $T_{\uparrow \downarrow}$ for the two orientations accrues from these four ellipses, which clearly indicates that the large spin-flip conductance is entirely due to the resonant surface states.

To understand the origin of the resonant spin-flip transmission, let us consider a metal with a surface band in the minority-spin channel which is orthogonal to the bulk states in both spin channels. Once we fix $\mathbf{k}_{\|}$, the problem becomes onedimensional (see Fig. 8). For a given $\mathbf{k}_{\|}$, the surface band is weakly coupled to the continuum of bulk Bloch states in its own spin channel. This coupling turns this band into a resonance and adds an imaginary self-energy (broadening) $\gamma_{0}$ to it. In addition, the surface band couples to the majority-spin bulk Bloch states via spinorbit interaction, which contributes $\gamma$ to its imaginary self-energy. One can identify two limiting cases. If $\gamma<\gamma_{0}$, the spin-orbit coupling acts as a weak perturbation of 


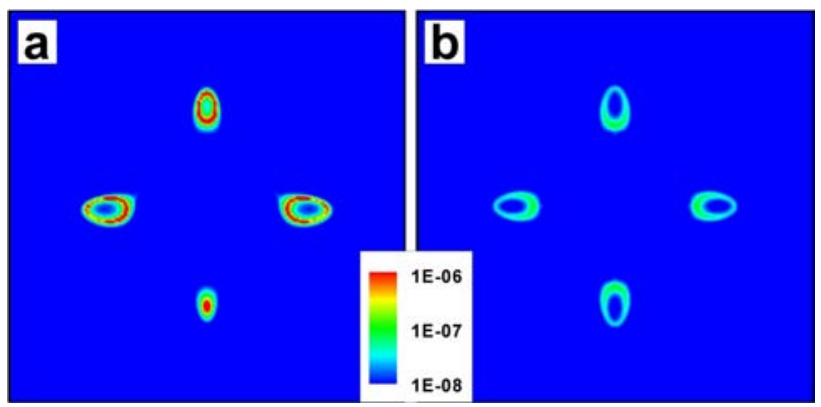

Fig. 7. Spin-flip transmission $T_{\uparrow \downarrow}$ for the in-plane (a) and normal-to-the-plane (b) magnetization at $E_{F}-0.102 \mathrm{eV}$.

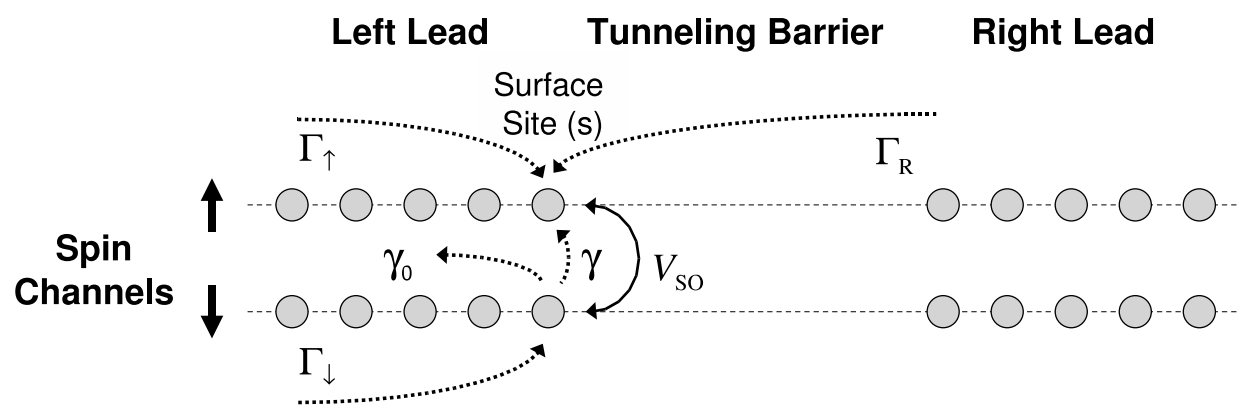

Fig. 8. Schematic illustration of the one-dimensional tight-binding model.

the minority-spin resonant surface band. It is obvious that in this case the spin-flip transmission across the barrier is small. On the other hand, if $\gamma>\gamma_{0}$, coupling to the minority-spin bulk states can be disregarded, and the surface band fully hybridizes with majority-spin bulk states (no matter how small $\gamma$ is compared to other energy scales, such as the bandwidth). In this limit, the bulk majority-spin state has a large minority-spin component at the surface in the energy range of the order $\gamma$ around $E_{s}\left(\mathbf{k}_{\|}\right)$, where $E_{s}$ is the energy of the surface band. Thus, there must be spin-flip transmission "hot spots" along the isoenergetic line $E=E_{s}\left(\mathbf{k}_{\|}\right)$ of the surface band wherever $\gamma>\gamma_{0}$ holds. Moreover, inside these hot spots, the coupling to bulk minority-spin states may be regarded as a weak perturbation, and therefore the minority spin-conserving transmission should be depressed, i.e. there should be an "antiresonance" in this channel.

To illustrate this effect in more detail, let us use the Green's function technique within a tight-binding model. Since the problem is effectively one-dimensional, consider a linear chain of sites terminating at the surface site labeled $s$. We treat the transmission problem by considering the surface atom as the active region, the remainder of the linear chain as the left electrode, and the other side of the barrier as the right electrode. We assume that the electronic structure of the barrier and the 
right electrode is spin-independent. Also, we assume that both the surface band and the bulk bands can be treated with a single-orbital basis. The character of these orbitals can not, of course, be s-type, but, for example, the surface band can be dominated by $d_{z z}$ character, while the majority-spin band may have $d_{x z}$ character at the given $\mathbf{k}_{\|}$point.

The transmission function $(2 \times 2$ matrix in spin indices $)$ then has the form:

$$
T_{\sigma \sigma^{\prime}}=\Gamma_{\sigma} g_{\sigma \sigma^{\prime}} \Gamma_{R} g_{\sigma^{\prime} \sigma}^{\dagger},
$$

where $g_{\sigma \sigma^{\prime}}$ is the full (retarded) Green's function of the surface site (surface Green's function), $\Gamma_{\sigma}=-2 \operatorname{Im} \Sigma_{\sigma}, \Sigma_{\sigma}$ is the spin-diagonal surface self-energy due to the coupling of the surface site to the left electrode, and $\Gamma_{R}$ is related in a similar way to the spin-independent self-energy due to the coupling to the right electrode across the barrier. All quantities in Eq. (19) depend on $\mathbf{k}_{\|}$.

We assume that the hopping across the barrier is small compared to all other energy scales including $\gamma$ and $\gamma_{0}$. (This limit can always be achieved by increasing the barrier thickness.) Formally, this means that $\Gamma_{R}$ can be neglected when the solution for $g_{\sigma \sigma^{\prime}}$ is sought and enters only explicitly through Eq. (19).

The spin-orbit interaction is treated as a perturbation acting at this surface site only. It has the following form:

$$
V_{S O}=\left(\begin{array}{cc}
0 & v \\
v^{*} & 0
\end{array}\right) \delta_{i s} \delta_{j s} .
$$

The unperturbed (retarded) Green's function $G^{(0)}$ of the entire system is spindiagonal. Taking the $\langle s|\ldots| s\rangle$ matrix element of both sides of the Dyson equation $G=G^{(0)}+G^{(0)} V_{S O} G$ leads to

$$
\begin{aligned}
& g_{\uparrow \uparrow}=g_{\uparrow \uparrow}^{(0)}+g_{\uparrow \uparrow}^{(0)} v g_{\downarrow \uparrow} \\
& g_{\uparrow \downarrow}=g_{\uparrow \uparrow}^{(0)} v g_{\downarrow \downarrow} \\
& g_{\downarrow \uparrow}=g_{\uparrow \downarrow} \frac{v^{*}}{v} \\
& g_{\downarrow \downarrow}=g_{\downarrow \downarrow}^{(0)}+g_{\downarrow \downarrow}^{(0)} v^{*} g_{\uparrow \downarrow},
\end{aligned}
$$

where $g_{\sigma \sigma^{\prime}}=\left\langle s\left|G_{\sigma \sigma^{\prime}}\right| s\right\rangle$ is the surface Green's function appearing in Eq. (19), and $g_{\sigma \sigma^{\prime}}^{(0)}$ the unperturbed surface Green's function. After some algebra, we find

$$
\begin{aligned}
& g_{\uparrow \uparrow}=\frac{1}{\left(g_{\uparrow \uparrow}^{0}\right)^{-1}-|v|^{2} g_{\downarrow \downarrow}^{0}} \\
& g_{\uparrow \downarrow}=\frac{v g_{\uparrow \uparrow}^{0}}{\left(g_{\downarrow \downarrow}^{0}\right)^{-1}-|v|^{2} g_{\uparrow \uparrow}^{0}} \\
& g_{\downarrow \uparrow}=\frac{v^{*} g_{\uparrow \uparrow}^{0}}{\left(g_{\downarrow \downarrow}^{0}\right)^{-1}-|v|^{2} g_{\uparrow \uparrow}^{0}}
\end{aligned}
$$




$$
g_{\downarrow \downarrow}=\frac{1}{\left(g_{\downarrow \downarrow}^{0}\right)^{-1}-|v|^{2} g_{\uparrow \uparrow}^{0}} .
$$

The unperturbed Green's function of the surface resonance may be represented as $g_{\downarrow \downarrow}^{0}=\left(E-E_{s}+i \gamma_{0}\right)^{-1}$. The term $|v|^{2} g_{\uparrow \uparrow}^{0}$ is the surface minority-spin selfenergy due to spin-orbit coupling; according to our prior definition, we have $\gamma=$ $-|v|^{2} \operatorname{Im} g_{\uparrow \uparrow}^{0}$. The majority-spin surface Green's function $g_{\uparrow \uparrow}^{0}$ is not strongly modified unless $|v|^{2} / \gamma_{0}$ is comparable to the majority band width.

In order to find $\Gamma_{\sigma}$ in Eq. (19), we recall that $\Sigma_{\uparrow}$ is simply the surface self-energy appearing in $g_{\uparrow \uparrow}^{0}$. Using the identity $\operatorname{Im} g_{\uparrow \uparrow}^{0}=\left|g_{\uparrow \uparrow}^{0}\right|^{2} \operatorname{Im} \Sigma_{\uparrow}$, we find

$$
\begin{aligned}
& T_{\uparrow \uparrow} \approx-2 \operatorname{Im} g_{\uparrow \uparrow}^{0} \Gamma_{R}, \\
& T_{\uparrow \downarrow}=\frac{2 \gamma \Gamma_{R}}{\left(E-E_{s}-\Delta\right)^{2}+\left(\gamma+\gamma_{0}\right)^{2}}, \\
& T_{\downarrow \downarrow}=\frac{2 \gamma_{0} \Gamma_{R}}{\left(E-E_{s}-\Delta\right)^{2}+\left(\gamma+\gamma_{0}\right)^{2}}, \\
& T_{\downarrow \uparrow}=\frac{\gamma_{0}}{\Sigma_{\uparrow}} T_{\uparrow \downarrow},
\end{aligned}
$$

where $\Delta=|v|^{2} \operatorname{Re} g_{\uparrow \uparrow}^{0}$ is the shift of the surface resonance.

First, we see that $T_{\downarrow \uparrow} \ll T_{\uparrow \downarrow}$ as long as the surface resonance is sharp enough $\left(\gamma_{0} \ll \Sigma_{\uparrow}\right)$. Further, if $\gamma \ll \gamma_{0}$, the transmission in the dominant spin-flip channel $T_{\uparrow \downarrow}$ is also small compared to $T_{\downarrow \downarrow}$. However, if $\gamma$ is comparable to $\gamma_{0}$, the $T_{\uparrow \downarrow}$ channel is comparable to $T_{\downarrow \downarrow}$. In fact, in our approximation the sum of these two channels depends only on $\gamma+\gamma_{0}$.

In order to find the resonant contribution of the given channel to the total conductance, we need to integrate over $\mathbf{k}_{\|}$. The resonant contribution is large only close to the surface band. Therefore we can first integrate normal to the curve $E=E_{s}\left(\mathbf{k}_{\|}\right)+\Delta\left(\mathbf{k}_{\|}\right)$, and then along this curve. We find

$$
\begin{aligned}
& T_{\uparrow \downarrow}(E) \propto N_{s}(E) \frac{\gamma}{\gamma_{0}+\gamma}, \\
& T_{\downarrow \downarrow}(E) \propto N_{s}(E) \frac{\gamma_{0}}{\gamma_{0}+\gamma},
\end{aligned}
$$

where $N_{s}(E)$ is the DOS of the surface band.

The results (33) agree very well with our first-principles calculations for $\mathrm{Fe}(001)$ surface. As was shown above, along the $\bar{\Gamma} \bar{X}$ lines, there is no mixing at all with the minority band when $v=0$. Near the bottom of the interface band, the surface band shrinks to four pockets along these lines (Fig. 7), and hence $\gamma_{0}$ is small for all the surface states at this energy. The relation $T_{\downarrow \uparrow} \ll T_{\uparrow \downarrow}$ is also confirmed by detailed calculations, as seen in Fig. 5.

The directional dependence of $T_{\uparrow \downarrow}$ (Fig. 7) comes from the relative magnitude of the spin-orbit contributions to $\gamma$ and $\gamma_{0}$. For the out-of-plane orientation, $\mathbf{M} \| \hat{\mathbf{z}}$, 
$V_{S O}$ mixes the interface states primarily to minority-spin bulk states, contributing mainly to $\gamma_{0}$. This spin-diagonal term is not included in the perturbation (20) but rather treated as a part of the unperturbed Hamiltonian. Therefore $\gamma \ll \gamma_{0}$, and the spin-flip transmission is small. For in-plane magnetization spin-orbit contributions to $\gamma_{0}$ and $\gamma$ are of the same order, and resonant spin-flip transmission sets in. Also, in agreement with our model, resonant spin-flip transmission occurs simultaneously with the reduction of the $T_{\downarrow \downarrow}$ component.

\section{4. $\mathrm{Fe} / \mathrm{GaAs}(001)$ interface}

To study spin-polarized transport across the Fe/GaAs(001) interface, we consider a $\mathrm{Fe} / \mathrm{GaAs} / \mathrm{Cu}(001)$ tunnel junction with a bcc $\mathrm{Cu}$ counter-electrode, which serves as a detector of spin polarization, in the spirit of Ref. 29. The bcc $\mathrm{Cu}$ electrode has a spin-independent free-electron-like band structure and a featureless surface transmission function, ${ }^{26}$ making it a perfect spin detector. This implies that variations in the spin polarization of the tunneling current with bias voltage found in the calculation performed for the $\mathrm{Fe} / \mathrm{GaAs} / \mathrm{Cu}(001)$ tunnel junction are entirely due to the changes in the spin transmission across the Fe/GaAs(001) interface. This makes the results of our calculations relevant to experiments. ${ }^{4,8,14}$

The particular junction studied consists of a semi-infinite Fe electrode, eight monolayers of GaAs barrier, and a semi-infinite bcc $\mathrm{Cu}$ electrode. We consider an As-terminated interface, motivated by the experiments on spin injection ${ }^{4,8}$ where the epitaxial $\mathrm{Fe} / \mathrm{GaAs}$ interfaces were grown in an As-rich environment. ${ }^{\mathrm{b}}$ Since intermixing of $\mathrm{Fe}$ and $\mathrm{As}$ atoms at this interface is not energetically favorable, ${ }^{31,32}$ we assume that the interface is abrupt. The small change of the As-Ga interplane distance of about $0.14 \AA$ due to relaxation ${ }^{32}$ is not taken into account.

Figures $9(\mathrm{a})$ and $9(\mathrm{~b})$ show the calculated local density of states (DOS) at the interface Fe monolayer and the integrated transmission as a function of energy for the $\mathrm{Fe} / \mathrm{GaAs} / \mathrm{Cu}$ junction. The energies are given with respect to $E_{F}$ which is found to be in the middle of the GaAs band gap in agreement with previous calculations. ${ }^{32,33}$ As is seen from Fig. 9(a), the minority spin dominates the interface DOS in the vicinity of the Fermi energy throughout the energy interval shown. There is a sharp peak in the minority-spin DOS between -50 and $-160 \mathrm{meV}$. The majority-spin transmission (Fig. 9(b)) exhibits a featureless free-electron-like energy dependence mirroring the featureless majority-spin DOS (Fig. 9(a)). In contrast, the minority-spin transmission is non-monotonic and dominates in two energy windows, between -130 and $-110 \mathrm{meV}$ and between +50 and $+175 \mathrm{meV}$ (Fig. 9b). The former local maximum corresponds to the sharp peak in the minority-spin interface DOS, whereas the latter maximum has no distinct analog in the DOS.

The energy dependence of the transmission is reflected in the voltage dependence of the spin-resolved current density shown in Fig. 9(c). It is seen that, while for

\footnotetext{
${ }^{\text {b}}$ Personal communication with S. A. Crooker.
} 

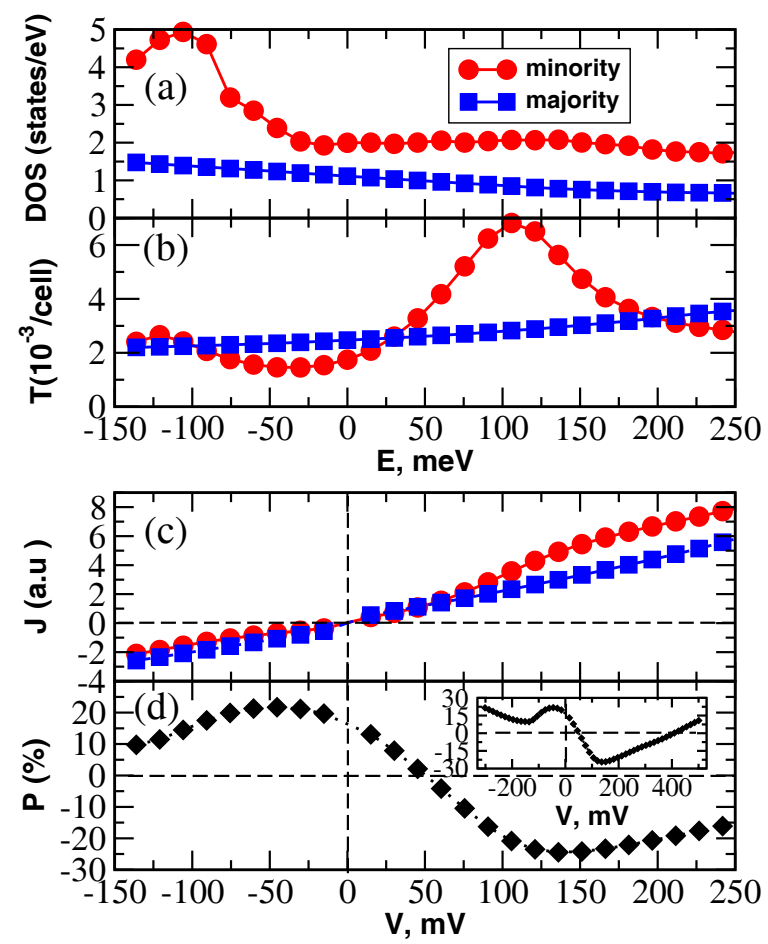

Fig. 9. Results of calculations for a Fe/GaAs/Cu tunnel junction: (a) spin-resolved local DOS for the interface Fe monolayer; (b) spin-resolved integrated transmission as a function of energy; (c) spin-resolved current density as a function of bias voltage; (d) spin polarization as a function of bias voltage. In (a) and (b), the Fermi level is set at zero energy.

negative bias voltages majority-spin electrons dominate the current density, there is a crossover at about $+50 \mathrm{mV}$ which makes the minority-spin current dominate at higher voltages up to $V=+400 \mathrm{mV}$ (see inset in Fig. 9(d)). This leads to the reversal of spin polarization at about $V=+50 \mathrm{mV}$ seen in Fig. 9(d). At $V=+400 \mathrm{mV}$, the spin polarization changes sign again reversing from anomalous (negative) to normal (positive). At $V=0$, we obtain high positive spin polarization similar to earlier work. ${ }^{34}$ The transmission peak between -130 and $-110 \mathrm{meV}$ (Fig. 9(b)) is too small to change the sign of the spin polarization and only leads to a reduction of the spin polarization by about $10 \%$. The reversal of the spin polarization with bias voltage is the central result of this study. In the following we will show that an interface resonant band is responsible for this anomalous behavior.

To discuss the non-monotonic bias dependence of the spin polarization, we focus on two energy intervals: the first around the small conductance peak at $-121 \mathrm{meV}$; and the second around the large conductance peak at $+106 \mathrm{meV}$. In Fig. 10 we show the minority-spin $k_{\|}$-resolved transmission for energies near these conductance peaks. The lower panels correspond to energies around the maximum of conductance in Fig. 9(a). Figure 10(H) corresponds to the energy of $+106 \mathrm{meV}$ approximately 


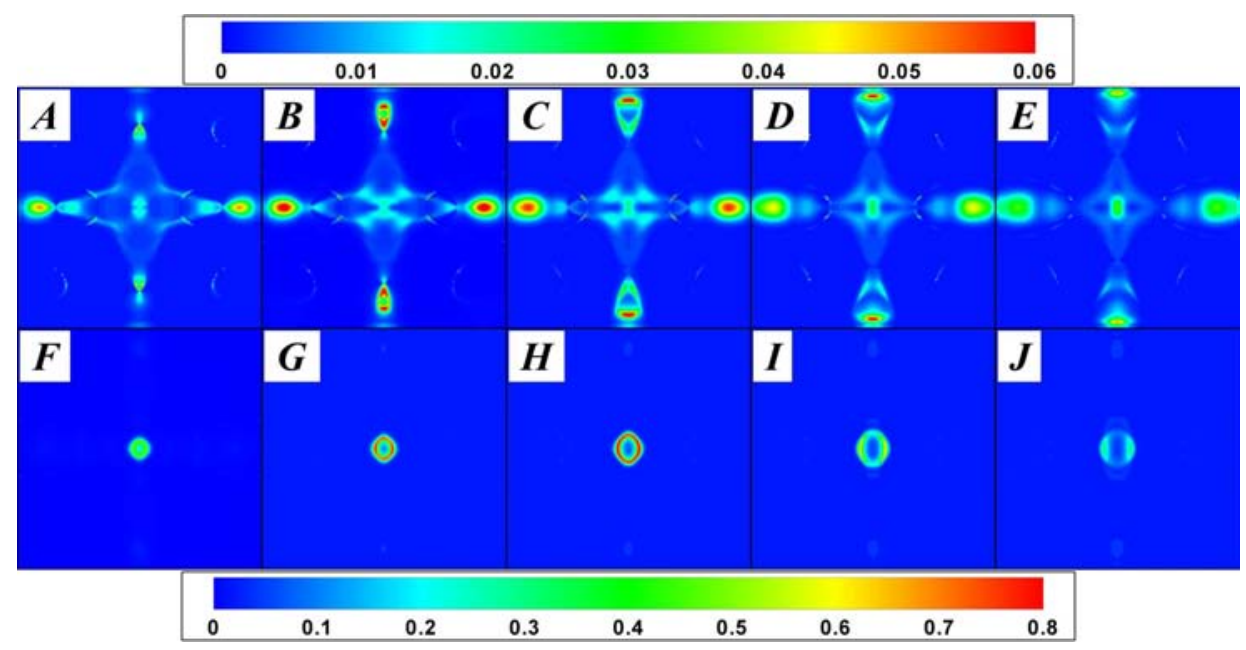

Fig. 10. Minority-spin transmission functions for the $\mathrm{Fe}(001)$ surface with a $\mathrm{Cu}$ counter electrode at various energies of interest. Figures are resolved by $k_{\|}$with abscissa along [100] and ordinate along [010]. The upper five panels are plotted for energies where the minority transmission has a small peak in Fig. 1(a)). From left to right, they correspond to the following energies $E-E_{F}$ : (A) $-151,(B)-136,(C)-121$ (local maximum of minority transmission), (D) -106, (E) $-91 \mathrm{meV}$. The lower half five panels are plotted for energies where the minority transmission is maximum in Fig. 1(a). From left to right they correspond to the following energies $E-E_{F}$ : (F) 45 , (G) 76 , (H) 106 (maximum of minority transmission), (I) 136, (J) $166 \mathrm{meV}$.

at the maximum of the peak. There is an oval closed loop of high transmission centered at the $\bar{\Gamma}$ point of the 2DBZ in all five panels. The upper five panels in Fig. 10 show the $k_{\|}$-resolved minority-spin transmission for energies around the large conductance peak. Figure 10(C) corresponds to the energy of $-121 \mathrm{meV}$ approximately at the maximum of the small peak. There are several narrow regions of high transmission around the boundaries of 2DBZ. In both cases, as we move away from the peak towards higher or lower energies, the intensity of the high transmission regions gradually decreases. This is the reason for the transmission peaks at $-121 \mathrm{meV}$ and $+106 \mathrm{meV}$. These bright features around the boundaries of $2 \mathrm{DBZ}$ and vicinity of $\bar{\Gamma}$ are caused by interface resonant bands. The transmission has $C_{2 v}$ symmetry which is the symmetry of the Fe/GaAs(001) interface. These bands are dominated by the minority-spin interface states arising from $d_{x^{2}-r^{2}}$ and $d_{x y}$ orbitals on interface Fe sites that couple with the bulk Fe $\Delta_{2^{\prime}}$ minority band.

In Fig. 11, we plot the $k_{\|}$-resolved minority-spin local Density of States (DOS) for layers of Fe near the interface. The lower three panels correspond to the energy of $+106 \mathrm{meV}$. Figure 11(D) is the $k_{\|}$-resolved DOS of Fe bulk, Fig. 11(E) is for an Fe subinterface layer and Fig. 11(F) for the Fe layer at the Fe/GaAs interface. Features similar to those seen in the transmission are present in the minority-spin DOS of the Fe interface layer. They are highly localized. As seen in Fig. 11(E), their intensity drops by a factor of five in the subinterface Fe layer. There is a clear difference 


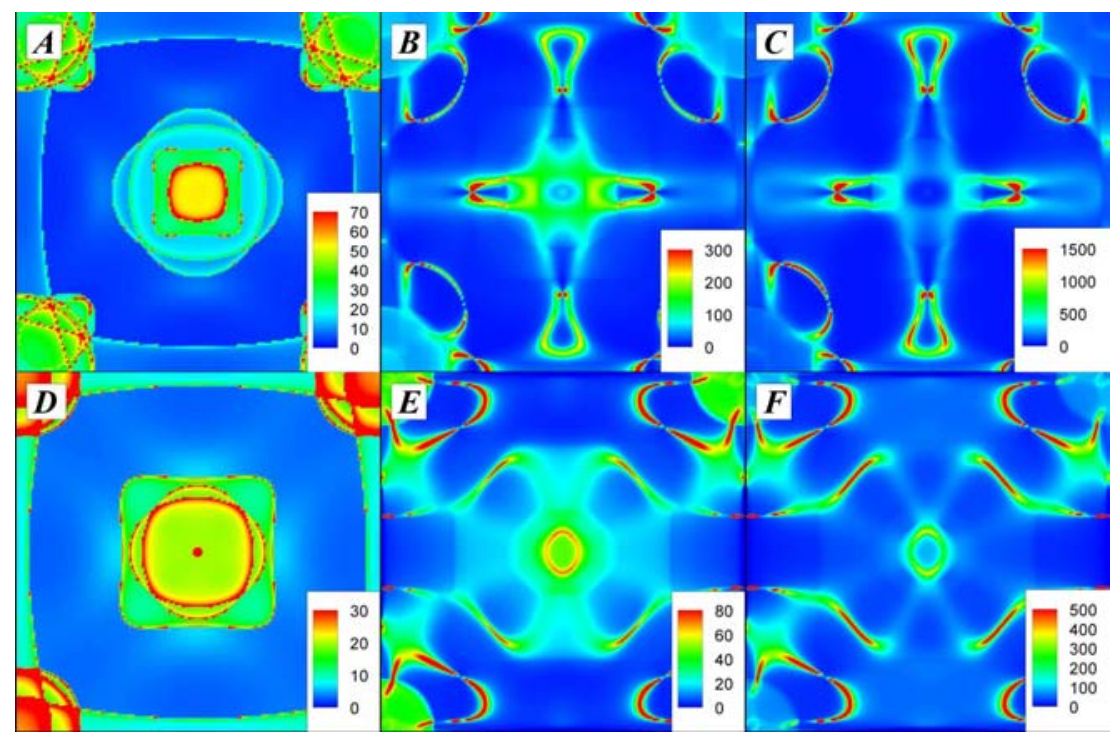

Fig. 11. Minority Fe DOS. The upper three panels are for $E-E_{F}=-121 \mathrm{meV}$ (local maximum of minority transmission) (A) bulk Fe, (B) subinterface Fe layer, (C) interface Fe layer. The lower three panels are for $E-E_{F}=106 \mathrm{meV}$ (maximum of the minority transmission) (D) bulk $\mathrm{Fe}$, (E) subinterface Fe layer, (F) interface Fe layer.

between the interface DOS along $[\overline{1} \overline{0}]$ and $[\overline{0} \overline{1}]$ directions. Those features are caused by interface states with the $\mathrm{C}_{2 \mathrm{v}}$ symmetry of the $\mathrm{Fe} / \mathrm{GaAs}(001)$ interface. The main difference between the interface DOS and the transmission is that in the second, there are additional high intensity features caused by interface states. This can be explained by the tunneling process in the GaAs. The tunneling of metallic real states through an insulating barrier is described in terms of the complex band structure in the barrier. Considering that $k_{\|}$is conserved during the transport through an ideal interface, for an electron entering the GaAs region with a real $k_{\|}$, the dispersion relation allows complex $k_{z}=q+i \kappa$. The wavefunction of the electron decays as $\sim e^{-\kappa z}$. Only complex bands with small decay parameter $\kappa>0$ are physically relevant. ${ }^{35}$ In Fig. 12, one can see that states in the middle of 2 DBZ along $\bar{\Gamma} \rightarrow \bar{L}$ and all symmetric directions have the largest $\kappa$ while states at the $\bar{\Gamma}$ point have the smallest. This is why after $8 \mathrm{ML}$ of GaAs the additional interface band crossings seen in interface DOS are invisible comparing to the dominant crossing in the vicinity of $\bar{\Gamma}$ point. In the upper three panels of Fig. 11, we show the $k_{\|}$-resolved minority-spin DOS for the energy of $-121 \mathrm{meV}$. We see again that features similar to those seen in the transmission are present in the minority-spin DOS of the Fe interface layer. The main difference is that in the interface DOS the features caused by interface states are located visibly closer to the $\Gamma$ point. This again can be explained by the tunneling process in the GaAs gap. In Fig. 2 of Ref. 35, one can see that along $\bar{\Gamma} \rightarrow \bar{X}$, states in the middle of the line have larger $\kappa$ than states around $\bar{X}$. The 


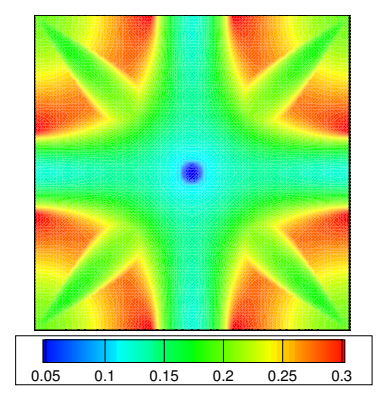

Fig. 12. A contour plot for the smallest decay parameter in the middle of GaAs gap. The scale is from 0.05 to $0.3 \mathrm{a} . \mathrm{u}$; the areas where the parameter is greater than 0.3 are blanked out. Clearly, there is a deep and narrow minimum at the $\Gamma$ point, and also a cross-shaped depression with another minimum at the $\bar{X}$ point.

tunneling process acts as a $k_{\|}$filter shifting the peaks seen in the interface DOS Fig. 11(C) towards $\bar{X}$ in the transmission Fig. 10(C).

The interface band at $-121 \mathrm{meV}$ has a complicated structure and crosses the 2DBZ at regions in the middle of the zone along $\bar{\Gamma} \rightarrow \bar{X}$ and symmetric directions. These states decay faster than the majority-spin states which are mainly concentrated around the $\bar{\Gamma}$ point. Therefore the interface band crossing at this energy will not play an important role in the spin polarization of the injected current. The interface band around $+106 \mathrm{meV}$ has a crossing in the vicinity of $\bar{\Gamma}$ in the $2 \mathrm{DBZ}$. From the sequence of energy cross sections presented in Fig. 10, it is seen that around $\bar{\Gamma}$ point, the band is parabolic and anisotropic with the interface $C_{2 v}$ symmetry. Because these states originate from the same region of the $2 \mathrm{DBZ}$ as the majority-spin states but have a much higher DOS, they dominate over the majority-spin transmission at this energy for any barrier thickness. For lower energies, the band barely crosses the Brillouin zone providing fewer states to the tunneling current while for higher energies the crossing occurs for larger $k_{\|}$. In both cases the contribution of the interface resonant band to the tunneling current is reduced. However, as seen in Fig. 11, it extends over a considerable energy window so that after energy integration, its contribution to the tunneling current is dominant for applied bias of more than $+50 \mathrm{mV}$, causing a reversal of spin polarization at $+50 \mathrm{mV}$. For sufficiently higher bias, outside the window presented in Fig. 11, the majority-spin current will become dominant again causing a second reversal of spin polarization.

In our calculations, the reversal of spin polarization occurs at positive applied bias, the corresponding current density results from an integration of $k_{\|}$-integrated transmission between $E_{F}$ and $E_{F}+\mathrm{eV}$. The transmitted electrons have energies above the Fermi level. In the experiments, ${ }^{4,8}$ this would correspond to electrons coming from GaAs into unoccupied states in Fe, that is forward applied bias (spin extraction). For the particular interface, no reversal of spin polarization can occur at negative bias, that is the experimental reverse bias (spin injection). The majority spin dominates the spin-polarized current in reverse bias and small forward 
bias whereas at a larger value of forward bias spin polarization reverses sign and minority spin dominates. This is in excellent agreement with one of the samples in Ref. 8 (sample C). Within our calculations, the bias value at which the polarization reverses its sign depends on the energy location of the interface resonance band near the $\bar{\Gamma}$ point. For the ideal interface examined here, this resonance band occurs at an energy slightly above $E_{F}$. However, diffusion of Fe atoms into GaAs as well as other parameters of the experimental growth can alter the electronic structure of the interface. Energy shifts of the interface band of the order of several tenths of an $\mathrm{eV}$ are quite possible. While we will not attempt here to predict all possible scenarios, it is very likely that for some of the experimental interfaces, the interface band crossing at the $\bar{\Gamma}$ point may occur below $E_{F}$; leading to reversal of spin polarization during injection of occupied Fe states into GaAs (reverse bias), such as in the case of sample B of Ref. 8. In conclusion, the bias dependence of spin polarization presented in Ref. 8 for sample B is very similar to that of sample C, the main difference is the point at which sign reversal occurs. Within our ab initio calculations, this point depends on the energy location of the interface band that crosses the $\bar{\Gamma}$ point of the $2 \mathrm{DBZ}$.

\section{Summary}

We have reviewed our recent findings about the influence of $\mathrm{Fe}(001)$ surface states on the spin-dependent transport of electrons across Fe surface and interface with GaAs. We have shown that Fe $3 d$ minority-spin surface states can be responsible for three effects: (a) tunneling anisotropic magnetoresistance; (b) strong variation of tunneling current spin polarization with applied bias; and (c) resonant spinflip scattering. So far, we have focused on the Fe(001) surface and Fe/GaAs(001) ideal abrupt interface. Our future studies include exploration of these effects in other important Fe interfaces (such as $\mathrm{Fe} / \mathrm{MgO}$ ) and a more detailed study of the $\mathrm{Fe} / \mathrm{GaAs}(001)$ interface which will include the investigation of how the stoichiometry and geometry of the interface can affect our main findings.

\section{Acknowledgment}

The work at Los Alamos National Laboratory was supported by the DOE Office of Basic Energy Sciences under Work Proposal Number 08SCPE973. K. Belashchenko is supported by the Nebraska Research Initiative and is a Cottrell Scholar of Research Corporation.

\section{References}

1. I. Zutic, J. Fabian and S. Das Sarma, Rev. Mod. Phys. 76 (2004) 323.

2. D. L. Smith and R. N. Silver, Phys. Rev. B 64 (2001) 045323.

3. E. I. Rashba, Phys. Rev. B 62 (2000) R16267.

4. S. A. Crooker, M. Furis, X. Lou, C. Adelman, D. L. Smith, C. J. Palmstrom and P. A. Crowell, Science 309 (2005) 5744.

5. A. T. Hanbicki, B. T. Jonker, G. Itskos, G. Kioseoglou and A. Petrou, Appl. Phys. Lett. 80 (2002) 1240. 
6. A. T. Hanbicki, O. M. J. van 't Erve, R. Magno, G. Kioseoglou, C. H. Li, B. T. Jonker, G. Itskos, R. Mallory, M. Yasar and A. Petrou, Appl. Phys. Lett. 82 (2003) 4092.

7. C. Adelmann, X. Lou, J. Strand, C. J. Palmstrom and P. A. Crowell, Phys. Rev. B 71 (2005) 121301.

8. X. Lou, C. Adelman, S. A. Crooker, E. S. Garlid, J. Zhang, K. S. M. Reddy, S. D. Flexner, C. J. Palmstrom and P. A. Crowell, Nat. Phys. 3 (2007) 197.

9. O. Krupin, G. Bihlmayer, K. Starke, S. Gorovikov, J. E. Prieto, K. Döbrich, S. Blügel and G. Kaindl, Phys. Rev. B 71 (2005) 201403.

10. S. Ohnishi, A. J. Freeman and M. Weinert, Phys. Rev. B 28 (1983) 6741.

11. P. D. Johnson, Y. Chang, N. B. Brookes and M. Weinert, J. Phys. Cond. Matter 10 (1998) 95.

12. J. A. Stroscio, D. T. Pierce, A. Davies, R. J. Celotta and M. Weinert, Phys. Rev. Lett. 75 (1995) 2960.

13. C. Gould, C. Ruster, T. Jungwirth, E. Girgis, G. M. Schott, R. Giraud, K. Brunner, G. Schmidt and L. W. Molenkamp, Phys. Rev. Lett. 93 (2004) 117203.

14. J. Moser, M. Zenger, C. Gerl, D. Schuh, R. Meier, P. Chen, G. Bayreuther, W. Wegscheider and D. Weiss, Appl. Phys. Lett. 89 (2006) 162106.

15. O. K. Andersen, Phys. Rev. B 12 (1975) 3060.

16. A. K. Rajagopal and J. Callaway, Phys. Rev. B 7 (1973) 1912.

17. I. V. Solovyev, A. B. Shick, V. P. Antropov, A. I. Liechtenstein, V. A. Gubanov and O. K. Andersen, Phys. Rev. B 31 (1989) 1285.

18. A. B. Shick, I. V. Solovyev, V. P. Antropov, A. I. Liechtenstein and V. P. Antropov, Phys. Metals Metallography 73 (1992) 41.

19. A. B. Shick, V. Drchal, J. Kudrnovsky and P. Weinberger, Phys. Rev. 54 (1996) 1610.

20. I. Turek, V. Drchal, J. Kudrnovsky, M. Sob and P. Weinberger, Electronic Structure of Disordered Alloy, Surfaces, and Interfaces (Kluwer Academic Publishers, Boston, 1997), p. 43.

21. I. V. Solovyev, A. I. Liechtenstein, V. A. Gubanov, V. P. Antropov and O. K. Andersen, Phys. Rev. 43 (1991) 14414.

22. O. Gunnarson, O. Jepsen and O. K. Andersen, Phys. Rev. B 27 (1983) 7144.

23. O. K. Andersen, Z. Pawlowska and O. Jepsen, Phys. Rev. B 34 (1986) 5253.

24. S. Datta, Electronic Transport in Mesoscopic Systems (Cambridge University Press, Cambridge, 1995), p. 117.

25. V. Popescou, H. Ebert, N. Papanikolaou, R. Zeller and P. H. Dederichs, Phys. Rev. B72 (2005) 184427.

26. K. D. Belashchenko, E. Y. Tsymbal, M. van Schilfgaarde, D. A. Stewart, I. I. Oleynik and S. S. Jaswal, Phys. Rev. B69 (2004) 174408.

27. M. N. Khan, J. Henk and P. Bruno, J. Phys. Cond. Matter 20 (2008) 155208.

28. B. Ackermann, R. Feder and E. Tamura, J. Phys. F: Met. Phys. 14 (1984) L173.

29. A. N. Chantis, K. D. Belashchenko, E. Y. Tsymbal and M. van Schilfgaarde, Phys. Rev. Lett. 98 (2007) 046601.

30. A. N. Chantis, K. D. Belashchenko, D. L. Smith, E. Y. Tsymbal, M. van Schilfgaarde and R. C. Albers, Phys. Rev. Lett. 99 (2007) 196603.

31. S. C. Erwin, S.-H. Lee and M. Scheffler, Phys. Rev. B 65 (2002) 205422.

32. D. O. Demchenko and A. Y. Liu, Phys. Rev. B 73 (2006) 115332.

33. W. H. Butler, X.-G. Zhang, X.-D. Wang, J. van Ek and J. M. MacLaren, J. Appl. Phys. 81 (1997) 5518.

34. O. Wunnicke, N. Papanikolaou, R. Zeller, P. H. Dederichs, V. Drchal and J. Kudrnovský, Phys. Rev. B 65 (2002) 064425. 
35. Ph. Mavropoulos, N. Papanikolaou and P. H. Dederichs, Phys. Rev. Lett. 85 (2000) 1088.

36. O. Krupin, G. Bihlmayer, K. Starke, S. Gorovikov, J. E. Prieto, K. Dobrich, S. Blugel and G. Kaindl, Phys. Rev. B 71 (2005) 201403.

37. L.-N. Tong, F. Matthes, M. Muller, C. M. Schneider and C.-G. Lee, Phys. Rev. B 77 (2008) 064421.

38. P.-J. Zermatten, G. Gaudin, G. Maris, M. Miron, A. Schuhl, C. Tiusan, F. Greullet and M. Hehn, Phys. Rev. B 78 (2008) 033301.

39. E. Y. Tsymbal, K. D. Belashchenko, J. Velev, S. S. Jaswal, M. van Schilfgaarde, I. I. Oleynik and D. A. Stewart, Prog. Mater. Science 52 (2007) 401.

40. K. D. Belashchenko, J. Velev and E. Y. Tsymbal, Phys. Rev. B 72 (2005) R140404.

41. A. N. Chantis, D. L. Smith, J. Fransson and A. V. Balatsky, arXiv: 0809.3456 (2008). 